\title{
Roadmap Towards Fusion Electricity (Editorial)
}

\author{
Antonius Johannes Donné ${ }^{1}$ (C)
}

Published online: 27 June 2019

(C) Springer Science+Business Media, LLC, part of Springer Nature 2019

There are many challenges on the path towards providing consumers with energy from fusion reactions (heat and electricity). Fusion works as it continuously happens in our Sun and in the stars, but realising the conditions to achieve fusion in a controlled way on Earth is not easy. The quest of fusion calls for progress in many different fields embracing amongst others plasma physics, materials, engineering, and neutronics. In Europe it was realised at the beginning of the present decade that in order to bring fusion electricity as quickly as possible to the grid, we do need to make a thorough plan, covering all the challenges that have to be tackled.

A first European Fusion Roadmap was published in 2012 by my predecessor Francesco Romanelli and his team. ${ }^{1}$ I often compare the roadmap with a navigation system, bringing someone as fast as possible from A to B. The navigation system evaluates all possible roads and then calculates the fastest/shortest/most scenic one. It even comes up with alternative routes in case there are obstructions or traffic jams. Similarly does the fusion roadmap. It tries to take into account that some research options might result into a show stopper, and therefore a fall-back solution needs to found in parallel. Since 2012 the European Fusion Roadmap has been used to prioritise all fusion research along eight main challenges, which will be described below. Only research that is aligned with the priorities of the roadmap is funded with European money, while other research-even though it is very interesting and can lead to high-impact scientific papers-is no longer funded by Europe. Research institutes and universities still have the option to pursue this research with funding from national sources. Looking back, it can be stated that since the introduction of the European Fusion Roadmap, fusion research in Europe has become much more coherent and goal-oriented by focusing on the development of the physics for ITER, the design of DEMO, the training of the next

\footnotetext{
$\triangle$ Antonius Johannes Donné

Tony.Donne@euro-fusion.org

1 EUROfusion, Garching, Germany
}

generation of physicists and engineers; all of these points with a well-defined time line. I am happy to see that some other countries have followed, by publishing their own fusion roadmaps.

Similar to a navigation system that needs to be updated at regular intervals, because roads have changed and new highways and bridges have been built, also the Fusion Roadmap needs updates to stay in line with internal and external developments. The release of the latest ITER assembly, operation and research plan was the main, but not the only, event that triggered Europe to publish a revised Fusion Roadmap by the end of $2018 .^{2}$ Other reasons where a newly adopted strategy to find alternative solutions for the plasma exhaust problems, the excellent results with Wendelstein $7-\mathrm{X}$, and new insights with respect to the design of the DEMO demonstration plant. The new edition is an evolution of the original Fusion Roadmap, keeping all the strong elements and the coherency. The central facilities on the roadmap are ITER, DEMO and the neutron-irradiation facility IFMIF-DONES (DEMO Oriented Neutron Source). ITER is well underway and will demonstrate that reaching the conditions for fusion on Earth is feasible. DEMO will show for the first time that electricity can be generated from the fusion process. DONES is needed to validate and qualify the new materials that are being developed to withstand the high neutron loads in DEMO and the future Fusion Power Plants.

The revised Fusion Roadmap has the Scientific and Technological Basis of the Fusion Power Plant as end point. This has been done to ascertain that a DEMO is designed in such a way that it can be extrapolated to a fusion reactor. If DEMO would be the end point of the roadmap, one would run the risk to make a design that delivers electricity at the earliest possible time, but that is not straightforward to extrapolate. In setting the high-level

\footnotetext{
${ }^{1}$ F. Romanelli et al., Fusion Electricity: a roadmap to the realisation of fusion energy, EFDA (2012) ISBN 978-3-00-040720-8.

2 A. J. H. Donné et al., European Research Roadmap to the realisation of fusion energy, EUROfusion (2018) ISBN 978-3-00061152-0.
} 
requirements for DEMO, stakeholders have been involved, including scientists and engineers from utilities, grid operators, nuclear power plants, nuclear waste, and safety. Their strong recommendation was to design for a DEMO between 300 and $500 \mathrm{MWe}$, as below $500 \mathrm{MWe}$ it is allowed to simply put the power on the grid; above this level one needs to get beforehand permission from the grid operators. Surprisingly, the stakeholders didn't consider it of the utmost importance to generate continuous electricity from fusion. Long-pulsed operation of the order of a few hours with a short dwell time would be also perfect, provided the pulse can be delivered reliably at the requested time. This is important given the high degree of intermittent sources in the grid.

The European Fusion Roadmap is depicted in the figure below. It doesn't have a clear timeline as the roadmap is event driven. Since ITER has published the expected dates for first plasma and for reaching full performance, it is possible to relate the schedule to an absolute time scale. The main facilities: ITER, DEMO and IFMIF-DONES are clearly depicted. conducting Divertor Test Tokamak in Italy, and the highfield COMPASS-Upgrade tokamak in Czech Republic. The work with all these devices is focused on finding regimes with high plasma performance while having benign conditions for the plasma facing components. For DEMO this implies that highly radiative plasmas have to be engineered in which much of the power is radiated away uniformly over the blanket surface, reducing the power load in the divertor. Of course much research needs to be done to develop so-called no-ELM regimes and to find techniques to reliably avoid or mitigate disruptions. In parallel much research is done to find more suitable divertor geometries (as snowflake, super-X, double-null divertors) and novel divertor materials (e.g. liquid-metal divertors). The specific challenge in DEMO will be not so much to limit the stationary heat load on the divertor to $5-10 \mathrm{MW} / \mathrm{m}^{2}$, but much more to limit that on the plasma-facing surfaces of the breeding blankets to values of the order of $1 \mathrm{MW} / \mathrm{m}^{2}$, as these need to be very thin to not hinder the neutrons on their way into the blanket. As can be seen in the diagram, ultimately also ITER will give input to DEMO. Addition-

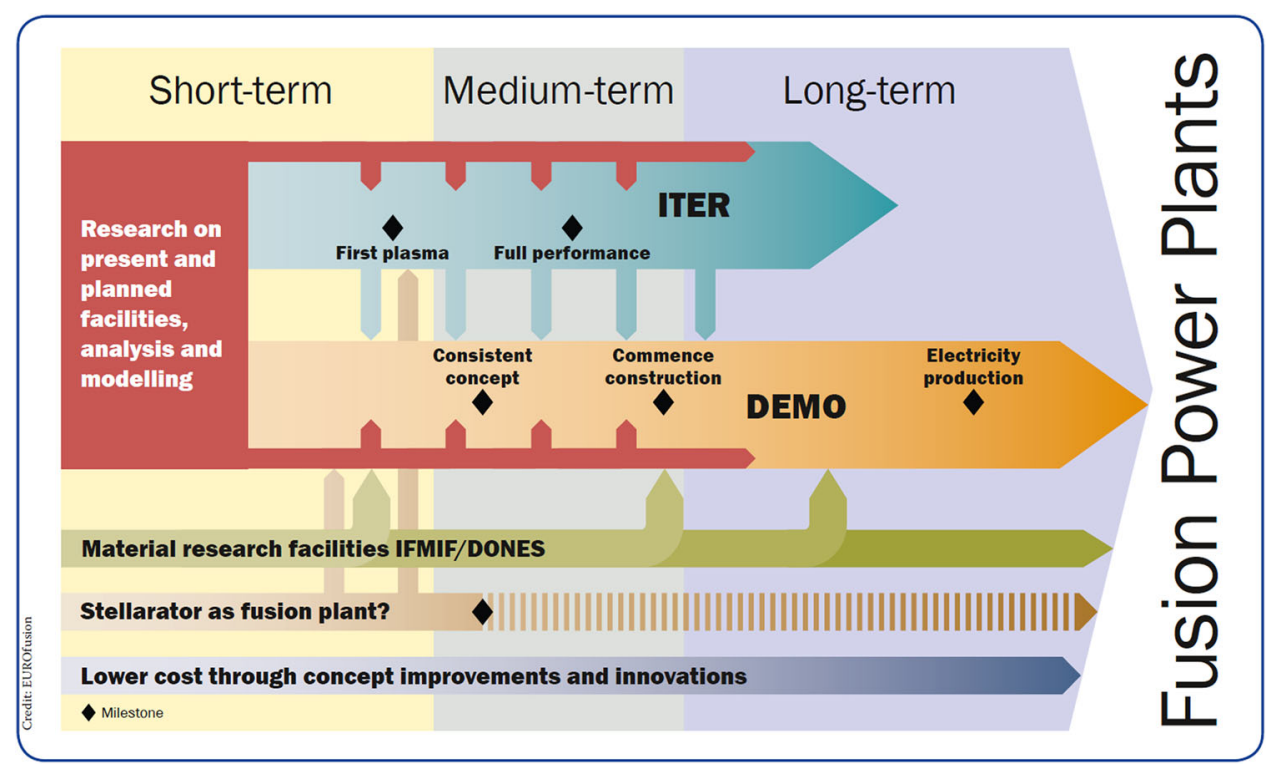

To make ITER and DEMO a success, much research is presently being done on a range of tokamaks: JET, the medium-sized tokamaks ASDEX-Upgrade, TCV and MAST-Upgrade for developing the ITER and DEMO physics basis and various facilities to test plasma-facing components: the WEST tokamak, the Magnum-PSI, PilotPSI and JULE-PSI. Two tokamak devices are presently being designed from national resources: the super- ally, plasma regimes need to be found that avoid the occurrence of transient heat loads due to Edge Localised Modes.

In Europe, the reference materials for the fusion reactor are EUROFER-97, a reduced-activation ferritic-martensitic steel alloy for the structural components, $\mathrm{CuCrZr}$ for the cooling channels and tungsten for the high heat-flux components. In principle one could build with the present 
materials a reactor that can withstand a neutron load of 20 dpa (displacements per atom). However, in DEMO and the future fusion power plant, the heat loads are more in the range of 50-100 dpa. Materials for this are being developed, and need to be tested and validated. Nowadays much of the materials testing is done in fission reactors, but for the components close to the plasma it is important to use a fusion-relevant spectrum of $14 \mathrm{MeV}$ neutrons. This is the purpose of IFMIF-DONES that is planned to be built early in the next decade near Granada in Spain.

Tritium breeding is another challenging topic. Various concepts are being studied and developed. ITER foresees the testing of six different Tritium Breeding Systems; some of them water-cooled, some others helium-cooled. The Test Blanket Modules (TBMs) either use ceramic breeders or molten lithium-lead. Europe is planning to test two TBMs in ITER: a helium-cooled pebble bed and a watercooled lithium-lead system. Work is also done on two other systems: helium-cooled lithium lead and doublecoolant lithium lead. The idea is that one of the two systems tested in ITER, will be used as starter blanket in DEMO; the second system might be utilised in a sector of DEMO as advanced blanket. Also DEMO will have the option to test some blanket options as specific modules.

It is especially the materials, but also the tritium breeding blankets that largely set the time path for DEMO. DEMO will be a nuclear device and to obtain the license to operate, all materials and systems need to be properly qualified. This will happen in DONES after 2030 for the materials, while the input on the test blanket modules will only come in after ITER starts DT operation in 2035. The European DEMO studies comprise the R\&D in all relevant fields, the design of all the nuclear components as well as the issue of licencing of the integrated device. ${ }^{3}$

The pre-conceptual design of the European DEMO is in full swing and the DEMO-team is preparing for a thorough gate review in 2020 to scrutinise all work which has been done thus far and, where possible to make choices for the design that will form the basis for the Conceptual Design Activity. The design on DEMO is carried out in a number of separate work packages, which closely interact with each other. The idea is to optimise the DEMO plant as a whole, instead of optimising individual components and then putting the together. This implies that special emphasis needs to be given to Key Design Integration

\footnotetext{
3 See for example Nuclear Fusion Reactors, D. Perrault, a report from IRSN by D. Perrault, https://www.irsn.fr/FR/Larecherche/publica tions-documentation/collection-ouvrages-IRSN/Documents/ITERVA_web_non_imprimable.pdf).
}

Issues that depend on several of the DEMO subcomponents.

The stellarator line in the European Fusion Roadmap is a long-term back-up strategy for the tokamak. At the moment of writing this editorial it is not clear whether we ever can engineer a tokamak control system that is disruption-free and ELM-free. In case we don't succeed in finding solutions for this, the stellarator might be an option. A stellarator doesn't suffer from disruptions and ELMs, but the design is technically much more challenging (think about the replacement and maintenance of tritium breeding blankets in the complex 3D geometry). The flagship stellarator in Europe is W7-X, which is now being equipped with an actively-cooled divertor system. Roughly at the end of next decade it needs to be decided whether one has enough confidence in the tokamak and, hence, whether it makes sense to continue with the stellarator line as a backup option.

The final arrow in the fusion roadmap schedule is related to the cost of electricity. At the end we do want to achieve a competitive price for fusion electricity. So much effort is devoted to work on innovations that help to reduce the price. This comprises amongst others work on high-temperature superconductors, that help to lower the cost of cryogenic cooling, work on remote handling to minimise the time needed for maintenance and replacements, virtual (or in silico) engineering to avoid laboratory testing of expensive components, additive manufacturing, etc.

There are many, and very diverse, challenges on the path to fusion electricity. Progress is needed in parallel in multiple fields. This editorial describes the European approach. Other Parties have different approaches. Any plan to realise electricity from a magnetically confined fusion plasma should take all above mentioned aspects into account, as in the end a nuclear reactor will be build that needs to be suitably tested and validated before the license to operate will be obtained from the nuclear regulator.

I am very pleased that many of the innovations that are necessary to bring fusion forward are described in this journal, ${ }^{4}$ which is one of the assets for anyone working in the field.

\section{May 2019}

Tony Donné

Publisher's Note Springer Nature remains neutral with regard to jurisdictional claims in published maps and institutional affiliations.

\footnotetext{
4 Journal of Fusion Energy, Special Issue: Creativity and Innovations on the Path to Fusion Energy Volume 38, Issue 1, February 2019.
} 